system for easy access to immunization and travel information. The new service provides answers to questions most frequently asked by healthcare professionals and parents.

The FAX materials contain comprehensive and up-to-date information regarding immunizations, including those needed for foreign travel. The system's information ranges from brief summaries suitable for the general public to the detailed ACIP statements for each vaccine.

Much of the material is designed to educate the general public on immunizations that protect children against preventable diseases. Healthcare workers and practitioners may wish to direct interested patients to the system for reference.

To contact the Immunization Voice Information System, call (404) 332-4554 for information specific to immunizations, and select the topic of interest and method of delivery. Or dial direct to the CDC FAX Information Service, (404) 332-4565 and request the immunization directory. For access to the CDC Voice Information System, dial (404) 332-4555 for entry into the CDC-wide voice system, which contains other topics in addition to immunization.

\section{Severe Isoniazid-Associated Hepatitis Identified in New York}

After the identification of a patient in New York who underwent liver transplantation because of severe hepatitis that developed during the use of isoniazid (INH) preventive therapy for latent tuberculous infection, an inquiry into other liver transplant centers in New York identified additional patients who had hepatitis attributed to INH.

Ten patients from New York were identified as being evaluated for liver transplantation because of severe acute hepatitis attributed to INH from January 1991 through May 1993. Of the 10 patients, one received both INH and rifampin. Another had received INH and discontinued its use one month before the onset of hepatitis symptoms because the patient had been exposed to tuberculosis resistant to INH and rifampin. Eight patients were taking INH alone (as therapy to prevent TB) at the onset of the hepatitis. The eight were aged 5 to 68 years; three were aged a20 years. Six were female. Because of the severity of illness, five of the eight patients received a liver transplant; one of these patients died after transplantation. The other three patients died while awaiting a donor liver.

The duration of INH use before the onset of hepatitis symptoms was either known or could be estimated for seven patients and ranged from 21 to 142 days; seven patients continued to take INH for at least 10 days after onset of symptoms. Initial symptoms of hepatitis included fatigue in five patients, nausea in five, abdominal pain in five, and anorexia in four. All patients had jaundice when they sought medical attention.

Previous reports have described cases of severe or fatal INH-associated hepatitis. One study estimated the risk for hepatitis as 20.7 per 1,000 persons with $4.6 \%$ of the cases being fatal. However, in this report neither the number of persons in New York who receive INH preventive therapy each year nor the number who have INH-associated hepatitis are known. Thus, it is unclear whether the number of patients in this report represents an increase in severe or lifethreatening INH-associated hepatitis or an improvement in the detection of the problem.

FROM: Severe isoniazid-associated hepatitis-New York, 1991-1993. MMWR 1993;42:545-547.

\section{Long-Term Acyclovir for Genital Herpes Is Safe and Effective}

The Acyclovir Study Group now has reported results through the fifth year of their ongoing long-term clinical trial.

After the first year of the study, all patients were offered open-label acyclovir in a dose of $400 \mathrm{mg}$ twice daily. Three hundred eighty-nine patients completed their fifth year of suppressive therapy and $25 \%$ of the patients had no recurrence for the entire five years. During each quarter of the fifth year, $86 \%$ to $90 \%$ of patients had no recurrence. The mean annual number of recurrences declined from 1.7 during the first year of the study to 0.8 during the fifth year of suppressive therapy. A small number of patients $(3.3 \%)$ needed a dose of $800 \mathrm{mg}$ acyclovir to suppress the outbreaks.

There were no significant changes in white blood cell counts, creatinine, or liver function tests in patients during the fifth year of treatment, and no cumulative toxicity was observed over time.

FROM: Goldberg LH, et al. Long-term suppression of recurrent genital herpes with acyclovir. A rch Dermatol 1993;129:582-587.

\section{MEDWatch Launched to Improve Adverse Events Reporting}

The U.S. Food and Drug Administration (FDA) recently announced a program to improve the safety of drugs, biologics, medical devices, infant formulas, dietary supplements, medical foods, and other regulated products by encouraging health professionals voluntarily to report any serious adverse events and product defects.

The new program, called MEDWatch, is a comprehensive approach to FDA's postmarketing surveillance, and is aimed at improving health professionals' involvement by focusing on their reporting.

"Physicians, nurses, and others who care for patients are the first to know when a drug or medical 